\title{
GIANT PULSE GENERATION USING GAIN MODULATOR*
}

\author{
I. Pinayev ${ }^{\dagger}$, V.N. Litvinenko, M. Emamian, N. Hower, M. Johnson, J. Patterson, G. Swift \\ FEL Lab, Duke University, Department of Physics, Durham, NC 27708, USA \\ S.H. Park, KAERI, 159 Deokjin-Dong Yusung-Ku, Taejon, Korea
}

\begin{abstract}
Using fast steering magnet allowed us to redistribute average power of a storage ring free electron laser in series of short pulses with high peak power. Theory of operation and experimental results are presented. Good agreement was found between measured and predicted parameters.
\end{abstract}

\section{THEORY OF OPERATION}

Average power of the free electron laser (FEL), installed on a storage ring, is limited by an induced energy spread caused by an interaction of an electron beam with optical pulse. This is a well-known Renieri limit $[1,2]$. Maximal average power is limited either by degradation of the FEL gain or by electron beam losses when energy spread exceeds storage ring acceptance. In most cases the first mechanism prevails and FEL demonstrates spiking behavior, well known in the conventional lasers [3].

To reach higher peak power it is possible to switch to a giant pulse mode, when all available in an active media power is released in single macropulse, followed by a relatively long relaxation period.

For the storage ring FEL conventional approach, utilizing modulation of optical cavity losses, is not suitable due to the insertion losses and high vacuum requirements. The active media - the electron beam - has negligible inertia and is much easier to manipulate. To stop lasing one can break synchronism between electron and optical bunches by modulating RF frequency (i.e. revolution frequency) or by moving the electron beam from the axis of the optical cavity. We prefer the latter method, because it allows faster handling of the electron beam and do not excite inevitable synchrotron oscillations. It also makes FEL pulse more reproducible with a higher peak power.

The gain modulator operates in the following manner:

- $\quad$ electron beam is shifted from the lasing orbit and is cooling off towards natural energy spread;

- when energy spread reduced to the desirable value, the electron beam is adiabatically moved to the lasing position;

- giant pulse is generated causing increase in the energy spread;

- cycle is repeated.

Frequencies of the betatron oscillations for the Duke storage ring [4] are $0.13 \cdot \mathrm{f}_{\mathrm{rev}}$ horizontal and $0.185 \cdot \mathrm{f}_{\mathrm{rev}}$ vertical, where $\mathrm{f}_{\text {rev }}=2.79 \mathrm{MHz}$ is a revolution frequency. Therefore the electron beam can be moved from one orbit

*This work is supported by DoD MFEL Program as administered by AFOSR.

†pinayev@fel.duke.edu to another without exciting of the betatron oscillations in few microseconds.

\section{GAIN MODULATOR DESIGN}

Local orbit distortion is usually created by a set of three or four steering magnets. However, we found it possible to employ a single magnet properly located on the storage ring. Before starting its design we formulated some requirements for the gain modulator:

- the electron beam moves in horizontal plane where vacuum chamber is significantly wider,

- displacement of the electron beam inside FEL region is preferable to be parallel to the optical axis,

- field integral should be sufficient to provide proper displacement of the electron beam at full energy of $1 \mathrm{GeV}$,

- in the lasing mode field in the gain modulator is set to zero,

- switching of the deflecting magnetic field should be done in few microseconds to provide "instant" turn on of the FEL,

- vacuum chamber should not increase broadband impedance, providing good coupling for the image currents.

The first two requirements defined the position of the gain modulator in the north straight section of the storage ring. The vacuum chamber has 4" diameter in this location, and due to the last requirement magnetic gap was chosen to be 6". Significant gap led to 8" length of the gain modulator to provide sufficient field integral.

\subsection{Vacuum chamber design}

The design of gain modulator vacuum chamber was the most challenging task due to contradiction in the requirements. From one side it should provide negligible impedance to the wake fields induced by the electron beam and from the other side it should suppress eddy currents, induced by the changing magnetic field. Two factors helped us to solve this problem: wake fields have much higher frequencies, and direction off eddy current is different from the electron bunch image current.

The final design of the vacuum chamber is shown in Fig. 1. 8" long glass cylinder with 5" inner diameter provides vacuum vessel. Cage of the aluminum rods with anodized ends couples wake currents induced by the electron beam, while providing high impedance for the eddy currents excited by the changing magnetic field. To prevent electrostatic charging on the inner surface of the glass tube thin chromium coating was applied. 

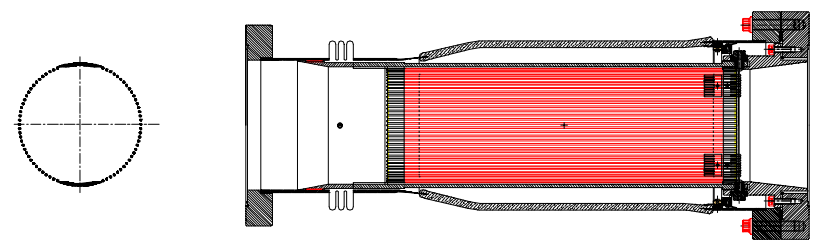

Fig. 1. Cross-section view of the vacuum chamber.

\subsection{Electrical design}

After the design of the vacuum chamber magnet construction was rather straightforward. With 6" gap in the gain modulator ferrite yoke with 60-turn coil rated to 10 A current provide field integral of $1440 \mathrm{Gs} \cdot \mathrm{cm}$.

High voltage MOSFET transistor serves as a switch of externally controlled current. The magnet inductance connected in parallel with clamping resistor and capacitor forms a critically damped contour with time constant of 8.8 microseconds. Added capacitor provides smooth transition without break in the first derivative. In this case the amplitude of residual oscillations proportional to the square of the product of the time constant and circular frequency of the betatron oscillations. Maximal repetition rate of $60 \mathrm{~Hz}$ is limited by the resistor wattage.

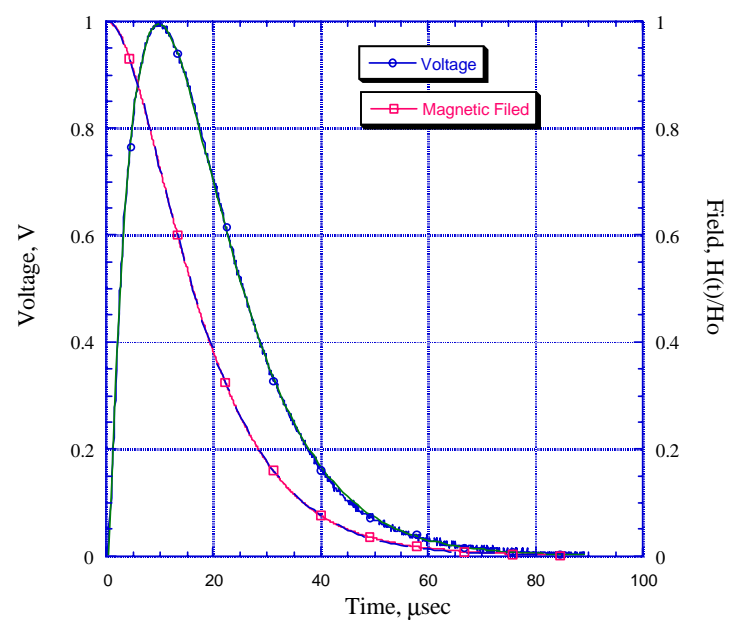

Fig. 2. Traces of voltage induced in a probe loop and corresponding magnetic field.

\section{GAIN MODULATOR PERFORMANCE}

After manufacturing and assembling we tested the gain modulator on the bench. The voltage induced in a pick-up coil by the decaying magnetic field was measured and integrated by digital oscilloscope LeCroy LC569A. The traces are shown in the Fig. 2. One can easily see that measured time constant is in a good agreement with calculated from the values of the components.

Next we determined the change in the equilibrium orbit of the real $500 \mathrm{MeV}$ electron beam when the gain modulator was excited with 4 A current. The displacement of the electron beam was $1 \mathrm{~mm}$ at the entrance to the OK4 and $2.5 \mathrm{~mm}$ at the end with the horizontal betatron tune of 0.11

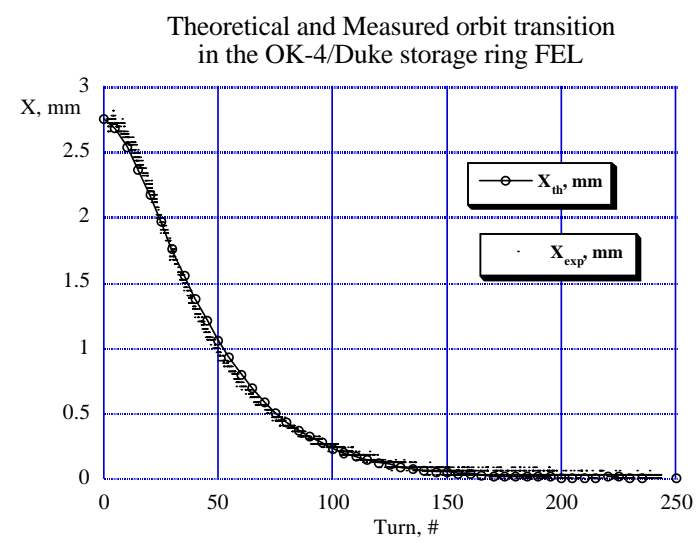

Fig. 3. Transition of the electron beam orbit

The dynamic characteristics of the gain modulator were obtained with the synchrotron radiation of the electron beam coming from a bending magnet. The light was focused on the S2044 position sensitive detector manufactured by Hamamatsu, which provides signal proportional to the displacement of the light spot. The oscilloscope trace showing the transition of the electron beam to the stationary orbit is depicted in the Fig. 3. No measurable betatron oscillations excited by the gain modulator were observed.

\section{GIANT PULSE GENERATION}

For a giant pulse operation of Duke storage ring FEL gain modulator periodically moves closed orbit of electron beam in the FEL region away from the axis of the optical cavity. This stops lasing and allows electron beam to reduce energy spread to the natural value. The fresh electron beam than is adiabatically returned to the optical axis and the FEL generates a giant pulse. The measurement of out-coupled optical power was done with a silicon diode to avoid problems with space charge. The photodiode signal comes through the second order lowpass filter which eliminates signal ripple associated with the temporal structure the FEL light. Small time constant of the filter, which equals to 0.3 microseconds, does not change the envelope of the giant pulse.

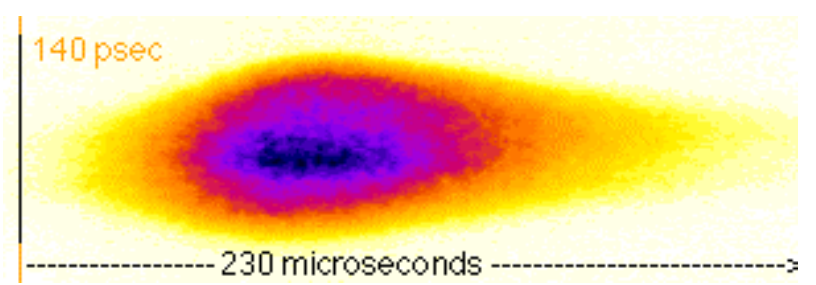

Fig. 4. Time structure of a giant pulse. 


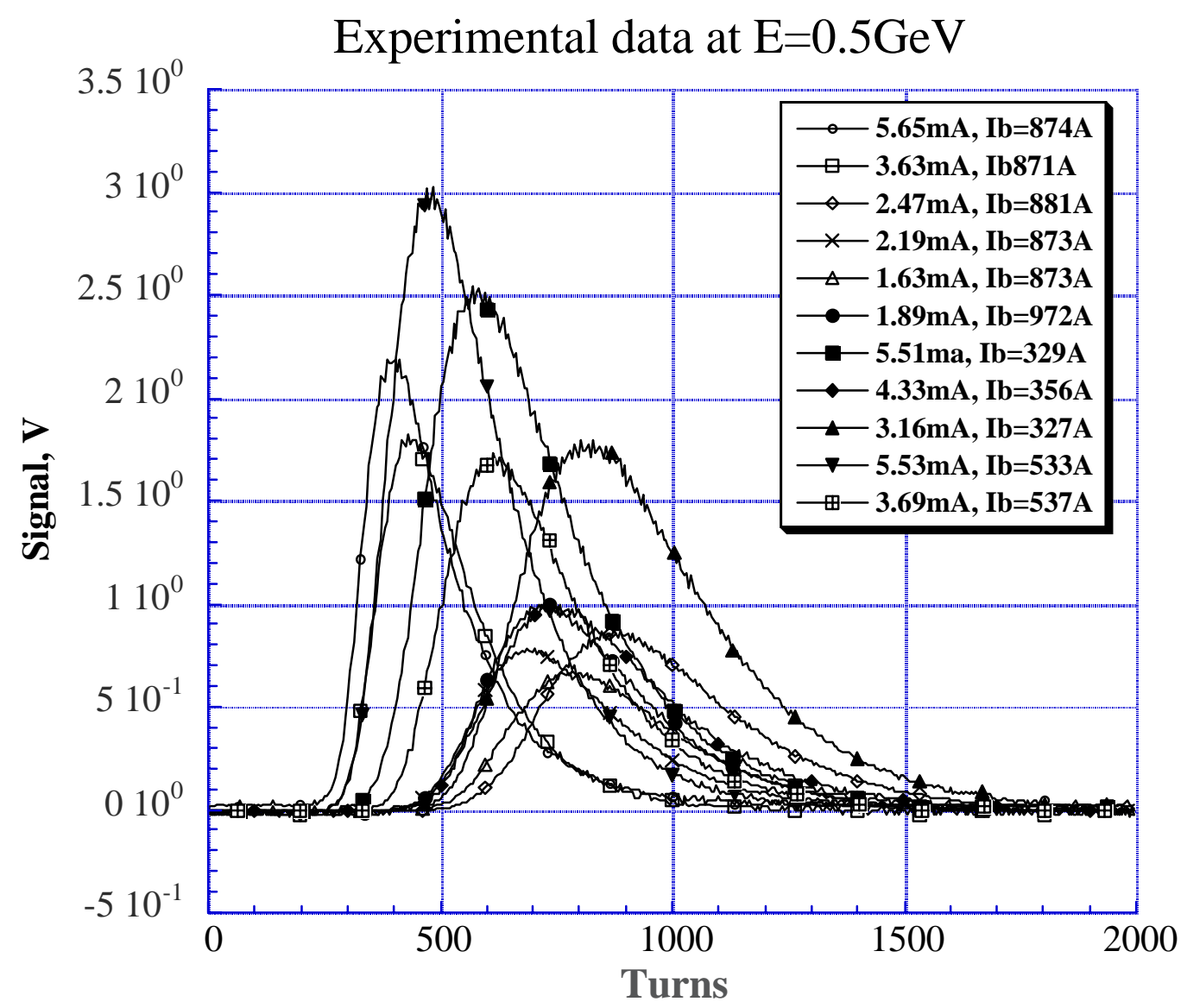

Fig. 5. Giant pulses measured with various electron beam and buncher currents.

The FEL lasing wavelength was $370 \mathrm{~nm}$ and electron energy was $500 \mathrm{MeV}$. After turning on gain modulator minor changes of the electron orbit in the OK-4/Duke FEL region were needed to maximize optical power. We attribute this to the slowly decaying fringe magnetic fields captured in the aluminum vacuum chamber adjacent to the gain modulator. Time structure of giant pulse obtained with the help of dual sweep streak camera is shown in Fig. 4. Comparison of the peak optical power of giant pulse with average value shows growth of peak power by three orders of magnitude, while long time average power is 2.3 times less.

The values of net gain of the OK-4/Duke FEL at different parameters found from the rise time constant are in a good agreement with estimations. Fig. 5 shows series of oscilloscope traces of optical power with different electron current and buncher settings. Using computer code [5] we modeled OK-4/Duke FEL behavior in the giant pulse mode. The perfect fit of the theoretical and experimental curves was found [6].

\section{CONCLUSION}

The gain modulator installed on the Duke storage ring allows us to generate giant pulses in stable and predictable manner. The performance of the OK-4/Duke FEL is well characterized and theoretical predictions have good agreement with experimental results.

\section{REFERENCES}

[1] N.A. Vinokurov and A.N. Skrinsky, "Power limit for an optical klystron installed on storage ring", Preprint INP 77-59, Novosibirsk, 1977.

[2] A. Renieri, Nuevo Cimento B 53 (1979) 160.

[3] O. Svelto, D.C. Hanna, Principles of Lasers, Perseus Publishing, 1998.

[4] V.N. Litvinenko, B. Burnham, J.M.J. Madey, S.H. Park, Y. Wu, "Duke storage ring UV/VUV FEL: staus and prospects", Nucl. Instr. and Meth., A375 (1996) 46.

[5] V.N. Litvinenko, B. Burnham, J.M.J. Madey, Y. Wu, Dynamics of the Duke storage ring UV FEL, NIM A358 (1995) 369.

[6] I.V. Pinayev, V.N. Litvinenko, S.H. Park, Y. Wu, M. Emamian, N. Hower, J. Patterson and G. Swift, Giant High-Peak Power Pulses in the UV OK-4/Duke Storage Ring FEL using the Gain Modulator, to be published in Nucl. Instr. and Meth. 